\title{
Stress-induced cardiomyopathy in the perioperative setting
}

\author{
Daniel Bainbridge, MD · Davy Cheng, MD
}

Published online: 28 April 2009

(c) Canadian Anesthesiologists' Society 2009

In their report in this issue of the Journal, Suk et al. describe the case of a 37-year-old female who developed anaphylaxis in response to cefotiam administration during a laparoscopic surgical procedure. ${ }^{1}$ A prolonged period of profound hypotension was followed by appropriate resuscitative maneuvers, but the patient subsequently developed a cardiomyopathy requiring ongoing medical treatment. The authors highlight a rather unusual presentation of Tako-Tsubo cardiomyopathy which occurred in the perioperative setting.

Increasingly, we are recognizing that Tako-Tsubo cardiomyopathy, also commonly referred to as stress-induced cardiomyopathy, transient cardiomyopathy, or apical ballooning syndrome, is a condition which may be more frequent in the perioperative setting than commonly appreciated. What is unusual about this case and somewhat unsettling is the observation that antibiotic-induced anaphylaxis or the treatment of anaphylaxis could, in turn, be associated with a potentially life-threatening cardiomyopathy. Interestingly, the pathophysiology of this cardiomyopathy may be similar to that of other disease entities that may be associated with acute cardiac dysfunction, including subarachnoid hemorrhage.

Based on the underlying etiology, stress-induced cardiomyopathy can be divided into two categories. The first category is related to emotional distress, leading to catecholamine surges and resulting in cardiac dysfunction. The second category is related to physical-pathological distress

\footnotetext{
D. Bainbridge, MD $(\bowtie) \cdot D$. Cheng, MD

Department of Anesthesia \& Perioperative Medicine, London Health Sciences Center-University Hospital \& St. Joseph's Health Care, University of Western Ontario, 339 Windermere Road, London, ON N6A 5A5, Canada

e-mail: daniel.bainbridge@lhsc.on.ca
}

secondary to disease entities, including pneumonia, severe acute asthma, or anaphylaxis, where catecholamine surges are secondary to the underlying disease process, which secondarily induces this unique cardiomyopathy. For both categories of stress-induced cardiomyopathy, there is an associated release of large quantities of epinephrine and norepinephrine, which are thought to be the primary mediators of the cardiomyopathy. The catecholamines temporarily disrupt the cardiac microvasculature resulting in myocardial dysfunction. In their report, Suk et al. ${ }^{1}$ infer that excess catecholamine surges may have occurred, either in response to the primary anaphylactic event or secondarily to the administration of exogenous epinephrine and norepinephrine given to treat the acute event.

The original report of stress-induced cardiomyopathy originates from Japan in $1991 .^{2}$ The authors reported on five cases from a series of 415 patients who presented with symptoms of acute myocardial infarction (AMI). Two of these patients had evidence of vasospasm at the time of angiography; two others developed vasospasm in response to the injection of ergonovine as a provocative test. There are numerous other reports in the literature of ambulatory patients presenting with stress-induced cardiomyopathy. The most comprehensive of these reports is from a systematic review of published case series. ${ }^{3}$ From the published series, it is inferred that up to $2 \%$ of patients presenting with AMI have a stress-induced cardiomyopathy, and furthermore, $27 \%$ of the presentations are secondary to emotional stress only, with $38 \%$ of patients having some type of physical stressor. The underlying causes in the remainder of cases were unreported. The associated mortality is reported to be $1.1 \% .^{3}$ While many case reports have documented an emotional trigger prior to the onset of symptoms, ${ }^{4-6}$ the few perioperative case reports have typically described events occurring intraoperatively under general anesthesia. ${ }^{7-9}$ 
Jayaraman et al. reported on the case of a 27-year-old female who developed signs consistent with an AMI complicated by pulmonary edema, which occurred within two minutes of establishing a pneumoperitoneum. ${ }^{7}$ Cabaton et al. reported a case of stress-induced cardiomyopathy following an anaphylactic reaction from succinylcholine. ${ }^{9}$ There are two case reports of this syndrome occurring outside the intraoperative period. Littlejohn reported on these two additional cases; one case involved a patient who experienced acute cardiomyopathy $4 \mathrm{~h}$ after electroconvulsive treatment therapy, and another case occurred during the pre-induction period within the operating room. ${ }^{8}$

The diagnosis of stress-induced cardiomyopathy is made on the basis of clinical, electrocardiograph (ECG), and echocardiographic findings; however, it is often difficult to differentiate this entity from an ischemic episode, and so, cardiac catheterization is often performed when the diagnosis is suspected. The term Tako-Tsubo is derived from the resemblance at cardiac catheterization of the left ventriculogram to a Tako-Tsubo pot (narrow neck with a broad base) used to catch octopi. The resemblance is formed when the basal segment of the heart contracts well (a distinguishing feature) while its apex is either hypo- or dyskinetic, ballooning outwards. The mid segments may or may not be involved. ${ }^{10}$ Bybee et al. suggest the use of four criteria to aid in the diagnosis of stress-induced cardiomyopathy: (i) transient akinesis or dyskinesis of the left ventricular apical and the mid-ventricular segment, with regional wall-motion abnormalities extending beyond a single epicardial vascular distribution; (ii) no angiographic evidence for plaque rupture or intracoronary thrombus formation; (iii) a new ECG abnormality (ST-segment elevation or T-wave inversion); and (iv) absence of recent head trauma, intracranial bleeding, pheochromocytoma, myocarditis, or hypertrophic cardiomyopathy. ${ }^{11}$ It should also be noted that the vast majority of cases reported in the literature involve women (95\%), most being postmenopausal, although the pathophysiology behind this association is not clear. There are known gender differences in the location and function of the cardiac sympathetic fibers. ${ }^{12}$

With the relatively new-found awareness of stressinduced cardiomyopathy comes the challenge of being able to accurately diagnose and treat this condition in the perioperative setting. Any patient who experiences severe physical or emotional reactions perioperatively and develops ECG abnormalities and intractable hypotension is at risk. Unfortunately, differentiating stress-induced cardiomyopathy from ischemic cardiomyopathy is challenging, and many patients often undergo coronary angiography when the diagnosis remains elusive. However, the wide- spread use of perioperative ultrasonography makes it likely that these patients will receive an echocardiogram in the perioperative setting, and anesthesiologists should appreciate the classic findings of this condition (a heart with normal basal function and a diminished dyskinetic apex, with or without mid-zone akinesis). Conversely, it should be remembered that patients with apparently normal left ventricles who experience a significant complication (anaphylaxis) may have markedly depressed cardiac function following the event.

The management of patients with stress-induced cardiomyopathy primarily involves supportive care. While the use of inotropes for vasoactive support was employed by Suk et al., ${ }^{1}$ some have suggested that the early implementation of balloon counter-pulsation, where available, may be superior, given that the etiology is likely secondary to catecholamine excess. ${ }^{3,12}$ The prognosis of stressinduced cardiomyopathy is generally favorable, reflecting the facts that the premorbid cardiac function is usually normal and the underlying precipitating cause (e.g., anaphylaxis, asthmatic attack) is usually transient. Complete resolution of stress-induced cardiomyopathy is typical. However, recurrences are possible, and patients should be warned that they may develop this condition again. The role for beta-antagonists for long-term prevention is unclear.

While some clinicians have speculated that the widespread use of angiography and perhaps echocardiography has led to greater recognition of Tako-Tsubo cardiomyopathy, it remains unclear why this condition remained unrecognized for so many years. The exact cause of this condition also remains a mystery. An animal model of immobilization stress, which generates ECG and echocardiographic findings similar to those found in humans who experience Tako-Tsubo cardiomyopathy, has been reproduced in rats. ${ }^{13}$ The observations from laboratory investigations suggest the sympathetic nervous system plays a primary role in contributing to this syndrome, which can be moderated experimentally by alpha and beta blockade. The laboratory model also demonstrates that estrogens may mitigate the changes, potentially conferring a cardioprotective effect.

Stress-induced cardiomyopathy is a disease entity which may clearly transcend the perioperative setting. As such, while we await consensus regarding standardized terminology for the variants of this unique cardiomyopathy and further information regarding the causes, mechanisms, and treatment, we now clearly recognize that stress-induced cardiomyopathy must be considered in the differential diagnosis of any patient presenting with signs or symptoms of an AMI in the perioperative period. 


\section{La cardiomyopathie provoquée par le stress dans un contexte périopératoire}

Dans leur compte-rendu publié dans ce numéro du Journal, Suk et coll. décrivent le cas d'une patiente de 37 ans ayant développé une réaction anaphylactique en réaction à l'administration de céfotiam pendant une intervention chirurgicale par laparoscopie. ${ }^{1}$ Une période prolongée d'hypotension profonde a été suivie par les manœuvres de réanimation adéquates, mais la patiente a par la suite manifesté une cardiomyopathie nécessitant un traitement médical continu. Les auteurs présentent une manifestation relativement peu fréquente de la cardiomyopathie de TakoTsubo survenant dans un contexte périopératoire.

Nous sommes de plus en plus conscients que la cardiomyopathie de Tako-Tsubo, aussi connue sous les noms de cardiomyopathie provoquée par le stress, cardiomyopathie transitoire ou syndrome de ballonisation apicale, est une affection peut-être plus fréquente qu'on ne le pense dans le contexte périopératoire. Ce qui est inhabituel et quelque peu troublant dans le cas présenté par Suk et coll. est l'observation qu'une anaphylaxie provoquée par les antibiotiques ou le traitement de cette anaphylaxie pourraient, eux aussi, être associés à une cardiomyopathie potentiellement fatale. Il est intéressant de noter que la physiopathologie de cette cardiomyopathie ressemble à celle d'autres entités morbides potentiellement associées à une dysfonction cardiaque aiguë, y compris une hémorragie sous-arachnoïdienne.

La cardiomyopathie provoquée par le stress peut être divisée en deux catégories selon son étiologie sous-jacente. La première catégorie est liée à une détresse émotionnelle provoquant des augmentations de catécholamines, ce qui cause une dysfonction cardiaque. La deuxième catégorie est liée à une détresse physiopathologique causée par des entités morbides, notamment la pneumonie, l'asthme aigu grave, ou l'anaphylaxie, où les augmentations de catécholamines sont secondaires au processus pathologique sous-jacent, lequel induit ensuite cette cardiomyopathie rare. Dans ces deux catégories de cardiomyopathies provoquées par le stress, une libération connexe de quantités importantes d'épinéphrine et de norépinéphrine survient, deux catécholamines qu'on pense être les médiateurs primaires de la cardiomyopathie. Les catécholamines désorganisent temporairement la microvasculature cardiaque, causant une dysfonction myocardique. Dans leur compterendu, Suk et coll. ${ }^{1}$ déduisent que des augmentations de catécholamines excessives sont survenues, soit en réponse à l'événement anaphylactique initial ou à la suite de l'administration d'épinéphrine et de norépinéphrine exogènes pour traiter la phase aiguë.
La première mention de cardiomyopathie provoquée par le stress a été faite au Japon en $1991 .^{2}$ Les auteurs ont rapporté cinq cas sur une série de 415 patients qui présentaient des symptômes d'infarctus aigu du myocarde (IAM). Deux de ces patients présentaient des signes appuyant l'hypothèse d'un vasospasme au moment de l'angiographie; deux autres ont manifesté un vasospasme en réaction à l'injection d'ergonovine comme test de provocation. La littérature contient nombre d'autres comptes-rendus de patients ambulatoires présentant une cardiomyopathie provoquée par le stress. Le plus complet de ces comptesrendus est une revue méthodique des séries de cas publiées. ${ }^{3}$ En se fondant sur les séries publiées, les auteurs de cette revue concluent que jusqu'à $2 \%$ des patients avec un IAM souffrent d'une cardiomyopathie provoquée par le stress, et que de plus, $27 \%$ des patients présentent des cardiomyopathies provoquées uniquement par un stress émotionnel et que $38 \%$ des patients présentent un type de déclencheur de stress physique. Les causes sous-jacentes dans les autres cas n'ont pas été rapportées. Les auteurs rapportent également que la mortalité associée est de $1,1 \%{ }^{3}$ Bien que dans nombre de présentations de cas un déclencheur émotionnel avant la survenue des symptômes ait été documenté, ${ }^{4-6}$ les quelques présentations de cas périopératoires décrivent en général les événements survenant dans la période peropératoire sous anesthésie générale. ${ }^{7-9}$ Jayaraman et coll. ont rapporté le cas d'une femme de 27 ans qui a manifesté des symptômes correspondant à un IAM compliqué par un œdème pulmonaire, lequel est survenu dans les deux minutes suivant l'apparition d'un pneumopéritoine. ${ }^{7} \mathrm{Cab}-$ aton et coll. ont rapporté un cas de cardiomyopathie provoquée par le stress à la suite d'une réaction anaphylactique à la succinylcholine. ${ }^{9}$ Deux présentations de cas de ce syndrome relatent des événements survenant hors de la période peropératoire. Littlejohn a rapporté ces deux cas supplémentaires; un cas impliquait un patient qui a manifesté une cardiomyopathie aiguë quatre heures après un traitement par électrochoc, et l'autre est survenu pendant la période de pré-induction dans la salle d'opération. ${ }^{8}$

Le diagnostic de cardiomyopathie provoquée par le stress est posé sur la base de résultats cliniques, électrocardiographiques (ECG) et échocardiographiques. Toutefois, il est souvent difficile de distinguer cette entité d'un épisode ischémique, c'est pourquoi un cathétérisme cardiaque est souvent réalisé lorsqu'un tel diagnostic est suspecté. Le terme de Tako-Tsubo provient de la ressemblance, lors du cathétérisme cardiaque, entre le ventriculogramme gauche et une urne Tako-Tsubo (goulot étroit et base large), utilisée pour capturer les poulpes. La ressemblance est visible lorsque le segment de base du cour se contracte bien (un trait distinctif) alors que l'apex est soit hypokinétique soit dyskinétique, bombant vers l'extérieur. Les segments médians peuvent ou non être impliqués. ${ }^{10}$ Bybee et coll. 
suggèrent l'utilisation de quatre critères pour aider à poser le diagnostic de cardiomyopathie provoquée par le stress: (i) akinésie ou dyskinésie transitoire du segment apical ventriculaire gauche et médian, accompagné d'anomalies régionales dans le mouvement des parois allant au-delà d'une distribution vasculaire épicardique unique; (ii) aucune évidence angiographique de rupture de plaque ou de formation de thrombus intracoronarien; (iii) une nouvelle anomalie à l'ECG (élévation du segment ST ou inversion de l'onde $\mathrm{T}$ ); et (iv) l'absence de traumatisme crânien, d'hémorragie intracrânienne, de phéochromocytome, de myocardite ou de cardiomyopathie hypertrophique récents. ${ }^{11} \mathrm{Il}$ est également important de souligner que la grande majorité des cas rapportés dans la littérature concernent des femmes (95\%), pour la plupart ménopausées, malgré le fait que la physiopathologie derrière cette association ne soit pas claire. Il existe des différences connues entre les sexes quant à l'emplacement et la fonction des fibers sympathiques cardiaques. ${ }^{12}$

La prise de conscience relativement nouvelle de l'existence d'une cardiomyopathie provoquée par le stress est accompagnée du défi de pouvoir diagnostiquer avec précision et traiter cette maladie dans un contexte périopératoire. Tout patient manifestant des réactions physiques ou émotionnelles graves pendant la période périopératoire et qui montre des anomalies à l'ECG et une hypotension réfractaire est à risque. Malheureusement, la distinction entre une cardiomyopathie provoquée par le stress et une cardiomyopathie ischémique est difficile à établir, et nombre de patients subissent une angiographie coronarienne lorsque le diagnostic est difficile à poser. Toutefois, l'utilisation répandue d'échoguidage périopératoire augmente la probabilité que ces patients reçoivent un échocardiogramme dans un contexte périopératoire, et les anesthésiologistes devraient détecter les résultats typiques de cette affection (un cœur avec une fonction basale normale et un apex dyskinétique et à la fonction réduite, avec ou sans akinésie de la zone médiane). En revanche, il faut également garder à l'esprit que les patients présentant un ventricule gauche apparemment normal mais subissant une complication importante (anaphylaxie) pourraient souffrir d'une fonction cardiaque considérablement altérée après l'événement.

La prise en charge de patients souffrant de cardiomyopathie provoquée par le stress implique principalement des soins de soutien. Suk et coll. ${ }^{1}$ ont utilisé des agents inotropes pour assurer le soutien vasoactif; toutefois, d'autres ont suggéré que la mise en place précoce d'un ballon intraaortique à contre-pulsion (lorsque cette technique est disponible) pourrait être plus efficace, étant donné que l'étiologie est probablement secondaire à un excès de catécholamines. ${ }^{3,12}$ Le pronostic d'une cardiomyopathie provoquée par le stress est en général favorable, étant donné que la fonction cardiaque prémorbide est en général normale et que la cause de précipitation sous-jacente à l'événement (par ex. anaphylaxie, crise d'asthme) est généralement transitoire. En règle générale, le rétablissement suite à une cardiomyopathie provoquée par le stress est complet. Toutefois, les rechutes sont possibles, et les patients doivent être informés qu'ils risquent de manifester cette affection à nouveau à l'avenir. Le rôle des bêta-antagonistes pour la prévention à long terme n'est pas clair.

Alors que certains cliniciens ont émis l'hypothèse que l'utilisation plus commune de l'angiographie et peut-être de l'échocardiographie a permis une reconnaissance plus importante des cas de cardiomyopathie de Tako-Tsubo, nous ne savons pas pourquoi cette affection est demeurée inconnue pendant de si longues années. La cause exacte de cette affection demeure elle aussi un mystère. Un modèle animal de stress d'immobilisation, qui génère des résultats d'ECG et échocardiographiques semblables à ceux que l'on retrouve chez les patients souffrant de cardiomyopathie de Tako-Tsubo, a été reproduit chez le rat. ${ }^{13}$ Les observations tirées de recherches en laboratoire suggèrent que le système nerveux sympathique joue un rôle important et contribue à ce syndrome, qui peut être atténué expérimentalement avec des alpha- et des bêta-bloquants. Le modèle de laboratoire démontre également que les oestrogènes pourraient atténuer les changements en conférant potentiellement un effet cardioprotecteur.

La cardiomyopathie provoquée par le stress est une entité morbide qui pourrait clairement transcender le contexte périopératoire. Dès lors, pendant que nous attendons un consensus concernant une terminologie normalisée pour les variantes de cette cardiomyopathie rare ainsi que des informations supplémentaires quant à ses causes, ses mécanismes et son traitement, nous reconnaissons dorénavant clairement que la cardiomyopathie provoquée par le stress doit être prise en compte dans le diagnostic différentiel de tout patient présentant des signes ou des symptômes d'IAM dans la période périopératoire.

Conflicts of interest The authors neither maintain nor are supported by any financial interests in commercial activity that may be associated with the content of this article.

\section{References}

1. Suk EH, Kim DH, Kweon TD, Na SW. Stress-induced cardiomyopathy following cephalosporin-induced anaphylactic shock during general anesthesia. Can J Anesth 2009; 56: 6. doi: 10.1007/s12630-009-9083-0.

2. Dote K, Sato H, Tateishi H, Uchida T, Ishihara M. Myocardial stunning due to simultaneous multivessel coronary spasms: a review of 5 cases (Japanese). J Cardiol 1991; 21: 203-14.

3. Gianni M, Dentali F, Grandi AM, Sumner G, Hiralal R, Lonn E. Apical ballooning syndrome or takotsubo cardiomyopathy: a systematic review. Eur Heart J 2006; 27: 1523-9. 
4. Biteker M, Duran NE, Civan HA, et al. Broken heart syndrome in a 17-year-old girl. Eur J Pediatr 2009. [Epub ahead of print].

5. Turley A, Graham R, Hall J. Takotsubo cardiomyopathy in two female patients: two case reports. Cases J 2008; 1: 325.

6. Parulekar P, Khawaja MZ, McWilliams ET. Chest pain after emotional and physical upset. BMJ 2008; 337: a107.

7. Jayaraman L, Sethi N, Sharma S, Gautam N, Sahai C, Sood J. Transient left ventricular apical ballooning post-pneumoperitoneum: Takotsubo cardiomyopathy. A case report. Minerva Anestesiol 2008. [Epub ahead of print].

8. Littlejohn FC, Syed O, Ornstein E, Connolly ES, Heyer EJ. Takotsubo cardiomyopathy associated with anesthesia: three case reports. Cases J 2008; 1: 227.

9. Cabaton J, Rondelet B, Gergele L, Besnard C, Piriou V. TakoTsubo syndrome after anaphylaxis caused by succinylcholine during general anaesthesia (French). Ann Fr Anesth Reanim 2008; 27: 854-7.
10. Kurowski V, Kaiser A, von Hof $K$, et al. Apical and midventricular transient left ventricular dysfunction syndrome (tako-tsubo cardiomyopathy): frequency, mechanisms, and prognosis. Chest 2007; 132: 809-16.

11. Bybee KA, Kara T, Prasad A, et al. Systematic review: transient left ventricular apical ballooning: a syndrome that mimics STsegment elevation myocardial infarction. Ann Intern Med 2004; 141: 858-65.

12. Wittstein IS, Thiemann DR, Lima JA, et al. Neurohumoral features of myocardial stunning due to sudden emotional stress. N Engl J Med 2005; 352: 539-48.

13. Ueyama T, Kasamatsu K, Hano T, Tsuruo Y, Ishikura F. Catecholamines and estrogen are involved in the pathogenesis of emotional stress-induced acute heart attack. Ann N Y Acad Sci 2008; 1148: 479-85. 